\title{
Avifauna del Caribe sur asociada a ecosistemas alterados en Limón, Costa Rica
}

\author{
Juan-Carlos Villegas Arguedas ${ }^{1} \&$ Jorge Villalobos Céspedes ${ }^{2}$ \\ 1. Dirección de Áreas Silvestres Protegidas, Área de Conservación Osa, Sistema Nacional de Áreas de Conservación, MINAE; \\ juan.villegas@sinac.go.cr \\ 2. Unidad de Estudios de Impacto Ambiental, Proyecto Hidroeléctrico Diquís, Instituto Costarricense de Electricidad; jvillalobosce@ice.go.cr
}

Recibido 14-I-2014 • Corregido 29-IV-2014 • Aceptado 2-VI-2014

\begin{abstract}
Southern Caribbean avifauna associated with altered ecosystems in Limón, Costa Rica. The Caribbean coast of Costa Rica, one of the areas with higher bird species richness in the country, is the main migratory route in Central America. We sampled the coast with visual counts (April-October 2002) and counted 1639 individuals of 123 species in 14 sample sites from Moin to Cahuita. The most common species were Psarocolius montezuma (Montezuma Oropendola), Ramphocelus passerinii (Passerini's Tanager) and Oryzoborus funereus (Thick-billed Seed-Finch). Most were in cattle land with isolated trees, followed by rivers and streams and the logged forest. Penshurt had the highest relative abundance, followed by Miravalles and Tuba Creek; the site with the lowest relative abundance was Tomatal. A cluster model indicated significant differences in avifauna composition. Pastures with trees, gardens, and mixed stands were the most diverse and had more birds. Vulnerable forest species occur in these forest remnants that are important for their conservation.
\end{abstract}

Key words: Birds, Caribe, Moín, Cahuíta, ornithology, species list, avifauna, Costa Rica.
Resumen: La costa caribeña de Costa Rica, una de las zonas con mayor riqueza de especies de aves en el país, es la ruta migratoria más importante en América Central. Muestreamos la costa con conteos visuales (abril-octubre 2012) y observamos 1639 individuos de 123 especies de aves en 14 sitios de muestreo de Moín a Cahuita. Las especies más comunes fueron Psarocolius montezuma (montezuma oropéndola), Ramphocelus passerinii (tangara de Passerini) y Oryzoborus funereus (semillero pico grueso). La mayoría se encuentran en los potreros con árboles aislados, luego en las orillas de los ríos y quebradas y también en los bosques intervenidos. Penshurt mostró la mayor abundancia relativa de las aves, seguido de Miravalles y Tuba Creek, y el sitio con la abundancia relativa más baja fue Tomatal. El modelo de conglomerados indica diferencias significativas en la composición avifaunistica. Repastos con árboles, jardines, y cultivos mixtos son los sitios más diversos y con mayor avistamiento de aves. Hay presencia de bosques con especies vulnerables que son importantes para la conservación.

Palabras clave: Aves, Caribe, Moín, Cahuíta, ornitología, listados, avifauna, Costa Rica.
Los constantes cambios en las matrices ecosistémicas por la deforestación y otros cambios en el uso del suelo, han promovido que la avifauna deba adaptarse a estos cambios y utilizar diferentes tipos de hábitats evolucionando a nuevas condiciones que éstos les dan, a pesar de la gran dependencia de este grupo faunístico de la calidad del hábitat (Cerezo, Robbins \& Dowell, 2009; Cepeda \& Montero, 2011).

Parte de estas adaptaciones son de comportamiento con utilización de sitios no habituales de alimentación o uso de nuevos recursos alimenticios y de refugio (Harris \& Pimm, 2004; Gawlik \& Crozier, 2007). No hay duda que la pérdida del bosque afecta negativamente a muchas especies especialistas de este ecosistema (Petit \& Petit, 2003; Lampila, Monkkonen \& Desrochers, 2005), no obstante recientes investigaciones sugieren que la conservación de matrices heterogéneas de hábitat favorecen la conservación de importantes poblaciones ornitológicas (Pereira \& Barrantes, 2009; Ramírez, 2010), siendo en ocasiones más importantes las zonas de ecotonos debido a la mayor concentración de la avifauna en dichos sitios entre áreas alteradas contiguas a bosques (Grundel \& Pavlovic, 2007).

El litoral Caribe de Costa Rica se reconoce como una de las zonas avifaunísticas con mayor riqueza de especies en el país, solo comparable con la riqueza que presenta el Pacífico Sur (Stiles \& Skutch, 1989), caracterizándose el Caribe por su importancia como ruta migratoria no solo en Costa Rica sino en toda Centroamérica, tanto para aves rapaces y carroñeras (Hidalgo, Sánchez, Sánchez \& Saborío, 1995) como para aves de menor tamaño como vireos, reinitas o bolseros. 
Muchos estudios de la avifauna del Caribe costarricense han tenido lugar en la región de La Selva en Sarapiquí (Stiles 1983, Sigel, Sherry \& Young, 2006), mientras que para el caribe sur los estudios sobre su avifauna se centran en sus corredores biológicos (ProAmbiente, 1998), las migraciones anuales de rapaces (Hidalgo et al., 1995) y la cuantificación de la cacería de aves canoras (Carbonell, Gastezzi \& Torrealba, 2004), no hay investigaciones que analicen este grupo faunístico en un tramo más amplio de la región Caribe (Stiles, 1983).

Este estudio pretende aportar información de la avifauna local y su relación con la matriz ecosistémica, a su vez aportar datos sobre la riqueza en especies en un ámbito geográfico muy extenso del Caribe sur de Costa Rica.

\section{MATERIALES Y MÉTODOS}

La investigación se desarrolló en la vertiente del Caribe costarricense, provincia de Limón donde se ubicaron catorce sitios de muestreo: Kent $\left(83^{\circ} 01^{\prime} \mathrm{W}, 9^{\circ} 54^{\prime} \mathrm{N}\right)$, Penshurt $\left(82^{\circ} 54^{\prime} \mathrm{W}, 9^{\circ} 47^{\prime} \mathrm{N}\right)$, Penshurt2 $\left(82^{\circ} 54^{\prime} \mathrm{W}, 9^{\circ} 46^{\prime} \mathrm{N}\right)$, Moín $\left(83^{\circ} 05^{\prime} \mathrm{W}, 9^{\circ} 59^{\prime} \mathrm{N}\right)$, Gibraltar $\left(82^{\circ} 53^{\prime} \mathrm{W}, 9^{\circ} 45^{\prime} \mathrm{N}\right)$, Finca Rosita $\left(82^{\circ} 59^{\prime} \mathrm{W}, 9^{\circ} 51^{\prime} \mathrm{N}\right)$, Río Tuba Creek $\left(82^{\circ} 54^{\prime} \mathrm{W}, 9^{\circ} 45^{\prime} \mathrm{N}\right)$, Santa Rosa $\left(83^{\circ} 03^{\prime} \mathrm{W}, 9^{\circ} 57^{\prime} \mathrm{N}\right)$, Bajos Tuba Creek $\left(82^{\circ} 53^{\prime} \mathrm{W}\right.$, $\left.9^{\circ} 44^{\prime} \mathrm{N}\right)$, Tomatal $\left(83^{\circ} 05^{\prime} \mathrm{W}, 9^{\circ} 57^{\prime} \mathrm{N}\right)$, Miravalles $\left(83^{\circ} 06^{\prime} \mathrm{W}\right.$, $\left.9^{\circ} 57^{\prime} \mathrm{N}\right)$, Río Congría $\left(82^{\circ} 58^{\prime} \mathrm{W}, 9^{\circ} 50^{\prime} \mathrm{N}\right)$, Miramar $\left(82^{\circ} 56^{\prime} \mathrm{W}\right.$, $\left.9^{\circ} 49^{\prime} \mathrm{N}\right)$ y Subestación Cahuíta $\left(82^{\circ} 52^{\prime} \mathrm{W}, 9^{\circ} 44^{\prime} \mathrm{N}\right)$ (Fig. 1).
Las visitas a los sitios se realizaron durante el año 2002 de abril a octubre, procurando recorrer el sitio al menos dos veces en un día de visita (mañana y tarde), cada sitio se visitó al menos en cuatro ocasiones (tres en la mañana y tres en la tarde); en cada uno de los catorce sitios se estableció un transepto de franja con ancho variable (30-50m) con el fin de detectar los individuos a ambos lados del trayecto (Bibby, Burguess \& Hill, 1992; Ralph et al., 1993; Chani et al., 1998), cada muestreo tuvo variantes en la longitud del recorrido debido a los accesos restringidos en algunas fincas y condiciones topográficas infranqueables, en promedio para todo el estudio en cada sitio se recorrió $5,6 \mathrm{~km}( \pm 500 \mathrm{~m})$. Los conteos se realizaron de 0600 a $1100 \mathrm{~h}$ y de las 1500 a las $1800 \mathrm{~h}$ debido a que como el registro fue de las aves observadas se requirió de luz día.

La obtención de los datos para especies e individuos por especies se recopilaron con búsqueda intensiva de las aves, registrando únicamente las observadas (Ralph et al., 1993; Bibby et al., 1992), no estableciendo ningún tiempo para este fin y registrando el hábitat en el cual se encontraron a las aves, mismos que se categorizaron en tipo y cobertura en el sitio de muestreo como: $\mathrm{Pa}$ (potrero arbolado) con una cobertura de $24,44 \%, \mathrm{Hr}$ (hábitat ribereño) con 13,33\% de cobertura, Bi (bosque intervenido) $22,22 \%$ de cobertura, Ca (cultivo arbolado) $4,44 \%$ de cobertura, $\mathrm{BiHr}$ (bosque intervenido con hábitats ribereños) $13,33 \%$ cobertura, $\mathrm{Cm}$ (cultivos mixtos) con $11,11 \%$

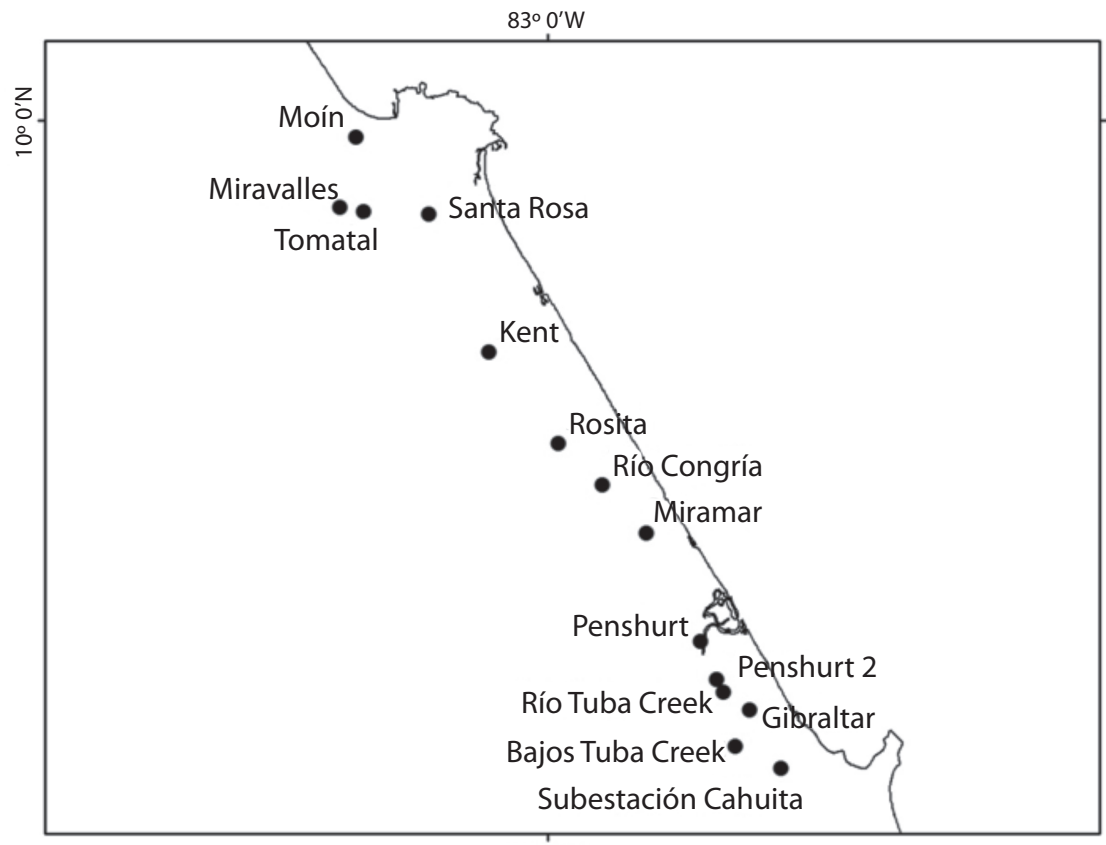

Fig. 1. Ubicación de los puntos de muestreo, Limón, Costa Rica. 
de cobertura, B (bosque maduro) 2,22\% de cobertura, LT (latizal tacotal) 4,44\% cobertura, P (potreros de gramíneas) con $2,22 \%$ de cobertura y LCB (latizal de caña brava Gynerium sagittatum) 2,22\% de cobertura.

Se determinó la riqueza de especies, abundancia relativa y distribución para cada especie; la riqueza de especies por hábitat se determinó con el tabulador de especies de Henderson y Seaby (2006) mediante su software Diversity, mismo que arroja el número exacto de especies que han sido registradas en una base de datos. La abundancia relativa se calculó dividiendo la cantidad de individuos cuantificados entre los kilómetros recorridos, (Bibby et al., 1992; Pereira \& Barrantes, 2009).

Para las comparaciones en la abundancia entre especies y sitios de muestreo se utilizó un análisis de varianza (ANOVA) no paramétrico Kruskall-Wallis con contrastes como prueba post-hoc (Zar, 1996). La similitud en la composición de especies se reflejó mediante el Índice de similitud de Jaccard sobre compartición de especies (Magurran, 1988), calculado con el software BioDap (Clay \&Thomas, 2000), el cual se utilizó para un análisis de conglomerados por sitios utilizando unión simple de la distancia euclidiana; para estos análisis se utilizó InfoStat 1.1 (Balzarini et al., 2002).

\section{RESULTADOS}

Se registró un total de 1639 individuos de 123 especies de aves entre los catorce sitios de muestreo a partir del recorrido de $78,5 \mathrm{~km}$ totales de muestreo en toda la región entre Moín y Cahuíta, donde las especie con mayores registros fueron Psarocolius montezuma (Lesson, 1830) (Montezuma Oropendola), Ramphocelus passerinii (Bonaparte, 1831) (Passerini's Tanager) y Oryzoborus funereus (Sclater, 1859) (Thick-billed Seed-Finch) (KruskallWallis $\mathrm{H}=162.25$, g.l.: $\left.122, \mathrm{p}<0.001^{* * *}\right)$.

No se sugieren diferencias estadísticamente significativas para los ensamblajes taxonómicos avifaunísticos entre los catorce sitios de muestreo (Kruskall-Wallis $H=13.64$, g.l: 13, $p=0.40$ ).

El hábitat de potrero arbolado $(\mathrm{Pa})$ fue el que presentó la mayor densidad de individuos con 31,70 individuos por este tipo de cobertura. El hábitat de cultivos mixtos tuvo una densidad de aves de 28,13 individuos, seguido del latizal-tacotal con 19,35 individuos para este tipo de cobertura (Fig. 2).

La riqueza de aves a lo largo de la zona estudiada ( $n=123$, Anexo 1), representa el $13,55 \%$ de las especies reportadas para el territorio nacional (Obando et al., 2013), el 32\% de las especies reportadas para el litoral caribe (Stiles, 1991) y el 65\% de las especies reportadas en la región Talamanca Caribe (ProAmbiente, 1998).

Los sitios que mostraron la mayor presencia de especies fueron Kent, Miramar y Moín, siendo en el caso de Kent el sitio con mayor cantidad de tipos de hábitats también; en estos tres sitios el hábitat que reporta la mayor riqueza de aves es el potrero arbolado $(\mathrm{Pa})$ con 27 especies. Estos mismos sitios con excepción de Moín reportan la menor riqueza de aves en el hábitat de bosque intervenido (Bi) con un registro de 2 especies. Moín por

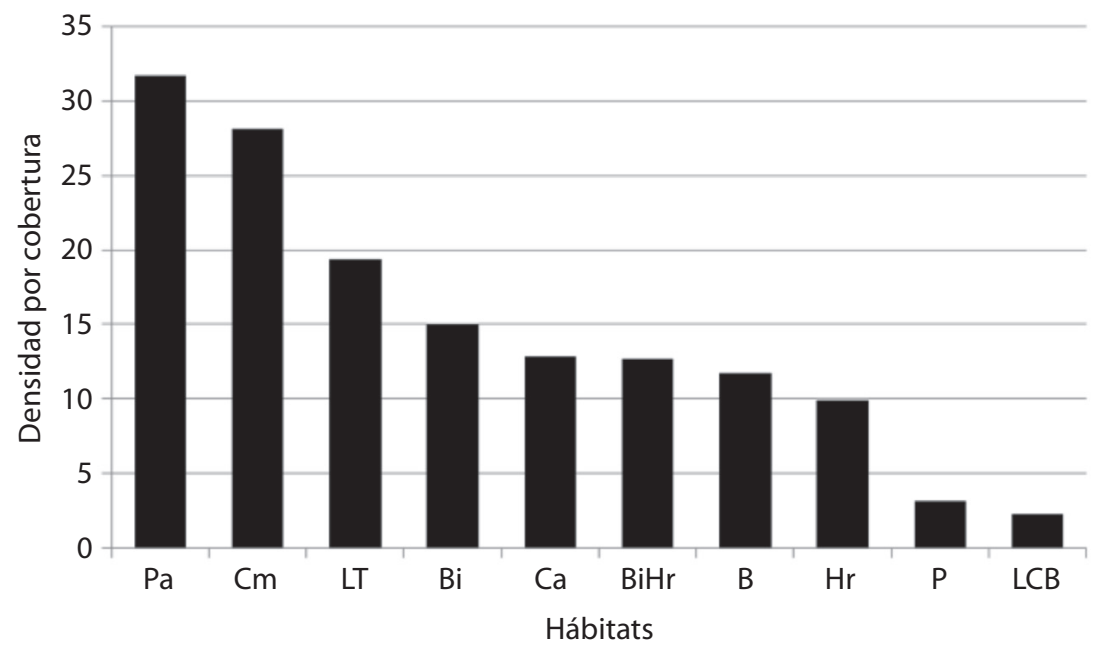

Fig. 2. Densidad de aves por tipo de hábitat, la comparación de medias por contrastes post-hoc es estadísticamente significativa para el Pa (Kruskall-Wallis H=35.56, g.l: 21, p<0.05). Tipos de hábitat: Pa (potrero arbolado), Cm (cultivos mixtos), LT (latizal tacotal), Bi (bosque intervenido), Ca (cultivo arbolado), BiHr (bosque intervenido con hábitats ribereños), B (bosque maduro), Hr (hábitat ribereño), P (potreros de gramíneas) y LCB (latizal de caña brava Gynerium sagittatum). 
CUADRO 1

Riqueza de especies de aves para cada uno de los sitios de muestreo y sus respectivos hábitats

\begin{tabular}{|c|c|c|c|c|c|c|c|c|c|c|}
\hline Sitio/Hábitat & B & $\mathrm{Bi}$ & $\mathrm{BiHr}$ & $\mathrm{Hr}$ & $\mathrm{Cm}$ & $\mathrm{Ca}$ & $\mathrm{Pa}$ & LT & LCB & $\mathrm{P}$ \\
\hline Kent & & 2 & 12 & 11 & 26 & & 27 & & 3 & 5 \\
\hline Miramar & 9 & 2 & 5 & & 15 & 12 & 27 & & & \\
\hline Moín & & 16 & 8 & & & & 30 & & & \\
\hline B. Tuba Creek & & 16 & & 18 & & & 18 & & & \\
\hline Río Congría & & 2 & 16 & 9 & & & 23 & 1 & & \\
\hline Tomatal & & 15 & & & & & 35 & & & \\
\hline Penshurt & & & 1 & 1 & & 36 & 6 & & & \\
\hline Miravalles & & 8 & & & & & 28 & & & \\
\hline Santa Rosa & & & 4 & 26 & & & 3 & & & \\
\hline Sub estación & 16 & & & & & & 17 & & & \\
\hline Río Tuba Creek & & & & 28 & 1 & & 2 & & & \\
\hline Finca Rosita & & 6 & & & & & & 19 & & \\
\hline Pens R. La Estrella & & 13 & & & 11 & & & & & \\
\hline Gibraltar & & 5 & & & 2 & & 16 & & & \\
\hline
\end{tabular}

su parte mostró la menor riqueza de especies en el hábitat de bosque intervenido con hábitat ribereño $(\mathrm{BIHr})$, con un total de 8 especies (Cuadro 1).

De acuerdo con el índice de abundancia relativa se tiene que Penshurt presentó la mayor cantidad de aves (IAR=38,67), seguida de Miravalles (IAR=32,75) y los bajos de Tuba Creek $(I A R=31,50)$, mientras que el sitio con la menor abundancia relativa es Tomatal $(I A R=14,33)$ (Cuadro 2).

El remplazo de especies fue alto entre los sitios sugiriendo la mayor similitud entre los sitios río Tuba Creek y Penshurt2 (60,6\% de similitud), seguidos de los sitios Penshurt2 y Gibraltar $(58,6 \%)$ y de Penshurt con bajos Tuba Creek (53,1\% de similitud), la finca Rosita presentó la avifauna mas diferenciada según el análisis de conglomerados por lo que se sugieren ensamblajes taxonómicos no muy similares para toda la región (Fig. 3).

Se reporta por primera vez en el caribe sur costarricense la observación de Dives dives (Deppe, 1839) (Melodious Blackbird) el día 17 de abril 2002 en un potrero arbolado rodeado de grandes extensiones de plantación de banano de Miramar de Limón, $2 \mathrm{~km}$ noroeste de Penshurt y $4 \mathrm{~km}$ noroeste de Cahuíta en un pie de monte de estribación de la Cordillera de Talamanca y de Sittasomus griseicapillus (Vieillot, 1818) (Olivaceous Woodcreeper) el 16 de mayo 2002 en un bosque intervenido en Moín de Limón, bosque protegido por JAPDEVA frente a la subestación eléctrica del Instituto Costarricense de Electricidad.
CUADRO 2

Índices de abundancia relativa para cada sitio visitado

\begin{tabular}{lccc}
\multicolumn{1}{c}{ Sitios } & $\begin{array}{c}\mathrm{N}^{\circ} \\
\text { individuos }\end{array}$ & $\begin{array}{c}\text { Esfuerzo muestreal } \\
\text { (km recorridos) }\end{array}$ & IAR \\
Penshurt & 174 & 4.5 & 38,67 \\
Miravalles & 131 & 4 & 32,75 \\
Bajos TubaCreek & 126 & 4 & 31,50 \\
Subestac. Cahuíta & 198 & 7,5 & 26,40 \\
Penshurt2 & 39 & 1,5 & 26,00 \\
Kent & 135 & 6 & 22,50 \\
Finca Rosita & 41 & 2 & 20,50 \\
Gibraltar & 38 & 2 & 19,00 \\
Miramar & 139 & 7,5 & 18,53 \\
Río TubaCreek & 70 & 4 & 17,50 \\
Moín & 198 & 12 & 16,50 \\
Santa Rosa & 74 & 4,5 & 16,44 \\
Río Congría & 61 & 4 & 15,25 \\
Tomatal & 215 & 15 & 14,33 \\
Total & 1639 & & \\
\hline
\end{tabular}

\section{DISCUSIÓN}

Diversos estudios han demostrado que la vertiente Atlántica de nuestro país posee una de las riquezas de especies de aves más altas, tanto de aves de interior de bosque como de hábitat alterados (ProAmbiente, 1998; Stiles, 1991). De manera lamentable la diversidad de aves 


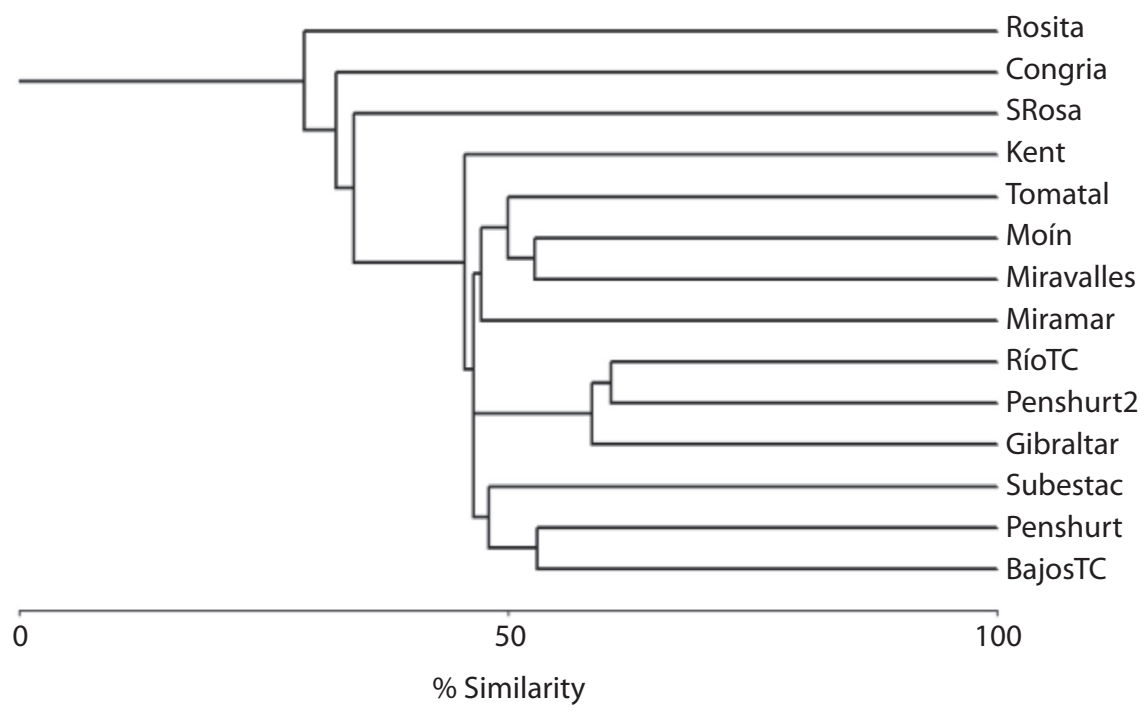

Fig. 3. Similitud de Jaccard en la composición de especies entre los sitios de muestreo (unión simple de la distancia euclidiana con un índice de correlación cofenética 0,88 )

de interior de bosque del Caribe costarricense se ha visto alterada y amenazada por un sin fin de actividades de origen antropogénico (Lopez, 1998), favoreciéndose a las especies cuyos requerimientos de hábitat son menos dependientes del bosque (Stiles \& Skutch, 1989); entre estas actividades se pueden citar la deforestación, las actividades silvoagropecuarias y la urbanización.

La fragmentación de las grandes masas de bosque provoca también que se fragmenten diferentes tipos de hábitat y microhábitat que son de gran importancia ecológica para la anidación, reproducción y alimentación de las diferentes especies de aves (Harris \& Pimm, 2004; Martin \& Mclntyre, 2007), impacto ambiental que afecta no solamente a las especies locales, sino también a las aves migratorias, mismas que en ocasiones presentan cambios en el comportamiento de forrajeo, estableciéndose en bordes de bosques (Rodewald \& Brittingham, 2007).

Distintas condiciones de las especies hacen que éstas sean más susceptibles que otras a la alteración de sus hábitats, entre éstas varias tienen características sociales como es el caso del perico frenteroja (Aratinga finschi Salvin, 1871) (Crimson-fronted Parakeet), el perico azteco (Aratinga nana Vigors, 1830) (Olive-throated Parakeet) y el chucuyo (Pionus senilis Spix, 1824) (White-crowned Parrot), condición importante durante su reproducción debido al requerimiento de sitios apropiados para nidificación y crianza de sus pichones; estas tres especies se han visto favorecidas por la heterogeneidad de hábitats en el Caribe sur costarricense, situación reconocida para muchas especies las cuales al fragmentarse los bosques $y$ establecerse mayor cantidad de tipos de hábitats aumentan sus abundancias locales (Donnelly \& Marzluff, 2004).

Otros como el martín pescador ventrirrufo (Chloroceryle inda Linnaeus 1766) (Green-and-rufous Kingfisher) tiene hábitos acuáticos en ríos cortos de poco caudal o el gavilán silbero o cangrejero (Buteogallus urubitinga Gmelin 1766) (Great Black-Hawk) que aprovecha las ramas de los árboles altos para acechar a sus presas, especialmente en bosques o limites de éstos, son vulnerables por la alteración de hábitats. El trepador delgado (Deconychura longicauda Pelzeln 1868) (Long-tailed Woodcreeper) y la gongolona (Tinamus major Gmelin 1789) (Great Tinamou) son especies que podrían verse mayormente perjudicadas de continuar la fragmentación de bosques en esta región sur del Caribe de Costa Rica debido a su gran dependencia de los ecosistemas boscosos para sus actividades, además por supuesto de las especies migratorias.

De acuerdo con el modelo de conglomerados es posible sugerir que a lo largo de la región evaluada no hay diferencias importantes en la composición avifaunística dada la similitud de hábitats de la que está compuesta la matriz ecosistémica, dominando los potreros arbolados, los jardines y los cultivos mixtos, además que la altitud de los sitios de muestreo fue similar (20-100m.s.n.m) impidiendo observar cambios altitudinales, los cuales se ha demostrado que si promueven más recambio en los ensamblajes taxonómicos (Stiles, 1983).

Únicamente las tres especies más abundantes (P. montezuma, R. passerinii y O. funereus) evidencian densidades 
superiores a 1 individuo por $\mathrm{km}$ recorrido, otras especies presentan densidades muy bajas, como Conopias albovittatus (Lawrence 1862) (White-ringed Flycatcher) , Baryphthengus martii (Spix, 1824) (Rufous Motmot), Cyanerpes cyaneus (Linnaeus 1766) (Red-legged Honeycreeper), Electron platyrhynchum (Leadbeater, 1829) (Broad-billed Motmot), S. griseicapillus (Vieillot, 1818) (Olivaceous Woodcreeper), Caryothraustes poliogaster (Du Bus de Gisignies, 1847) (Black-faced Grosbeak) o Carpodectes nitidus (Salvin, 1865) (Snowy Cotinga) haciendo de estas especies (esta última endémica de la región) las más vulnerables por fragmentación de bosques o alteración de sus hábitats.

De manera similar a lo reportado por Pereira y Barrantes (2009) para la zona sur de Costa Rica, el resto de la avifauna en la región Caribe sur en Limón se distribuye de manera continua posiblemente por la dominancia de ecosistemas alterados como los potreros arbolados o los cultivos mixtos, con discontinuidad solo en las especies de bosque como se mencionó.

El reporte para el caribe sur de Costa Rica de la observación de un individuo de la especie $D$. dives (Deppe, 1839) (tordo cantor) en la comunidad de Miramar de Limón podría considerarse el primer avistamiento de esta especie para esta región del país, no obstante previo a este reporte se ha registrado la ampliación de distribución de esta especie, particularmente en el pacífico central (Sánchez, Naoki \& Zook, 1998) sugiriendo su avance asociado a la ampliación de la frontera agrícola en todo el país (Stiles \& Skutch, 1989), también se reporta S. griseicapillus en Moín de Limón, lo que reafirma a la especie como local en el caribe, aunque poco común según Stiles y Skutch (1989).

La avifauna de esta región sur del Caribe costarricense no es similar a lo largo de esta vertiente, con diferencias en su distribución y con algunas pocas especies muy abundantes, particularmente las aves del Caribe sur están asociadas a ecosistemas alterados por el hombre como los potreros con árboles, los cultivos variados, los jardines, los tacotales y en menor grado a los remanentes de bosques intervenidos. Con este estudio se aportan datos de una gran región geográfica de Costa Rica y se compara su diversidad a lo largo de ésta, además se sugieren dos ampliaciones de distribución de dos especies observadas durante el mismo.

\section{AGRADECIMIENTOS}

A Luz Marina Rodríguez y Alexander Madrigal por su colaboración en algunas de las visitas de campo e identificación de varias especies, y en general a todo el personal del proyecto de reconstrucción de la línea de transmisión eléctrica entre Moín y Cahuíta del Instituto Costarricense de Electricidad.

\section{REFERENCIAS}

Balzarini, M. G., Casanoves F., Di Rienzo, J. A., González, L. A., Robledo, C. W., \& Tablada, E. M. (2002). InfoStat, versión 1,1, Manual del Usuario. Grupo InfoStat, FCA, Universidad Nacional de Córdoba. (1 a.ed.). Editorial Brujas: Córdoba, Argentina.

Bibby, C. J., Burguess, N. D., \& Hill, D. A. (1992). Bird census techniques. Academic: London, United Kingdom.

Carbonell, F., Gastezzi, P., \& Torrealba, I. (2004). Cacería de aves en Siquirres-Matina y Limón. ACLA-C, INBio: Heredia, Costa Rica.

Cepeda, M. F., \& Montero, J. L. (2011). Variación en la Composición y Abundancia de Aves Terrestres en la Reserva de la Biosfera Ría Lagartos, Yucatán, México. Mesoamericana, 15(2), 109.

Cerezo, A., Robbins, C., \& Dowell, B. (2009). Uso de habitats modificados por aves dependientes de bosque tropical en la región caribeña de Guatemala. Rev. Biol. Trop, 57(1-2), 401-419.

Chani, J. M.; Bucher, E.; Echeverría, A.; Marigliano, N. L., \& Brandán, Z. J. (1998).

Comparación entre censos de punto y recorrida en una comunidad de aves del bosque chaqueño. Vida Silvestre Neotrópical, 7(23).

Clay, D., \& Thomas, G. (2000). BIO-DAP. Ecological diversity and its measurement. Resource Conservation, Fundy National Park, Alma New Brunswick, Canada.

Donnelly, R., \& Marzluff, J. M. (2004). Importance of reserve size and landscape context to urban bird conservation. Conservation Biology, 18(3), 733-745.

Gawlik, D. E., \& Crozier, G. E. (2007). A test of cues affecting habitat selection by wading birds. The Auk, 124(3), 1075-1082.

Grundel, R., \& Pavlovic N. B. (2007). Distintiveness, use, and value of Midwestern oak savannas and woodlands as avian habitats. The Auk, 124(3), 969-985.

Harris, G. M., \& Pimm, S. L. (2004). Bird Species' Tolerance of Secondary Forest Habitats and Its Effects on Extinction. Conservation Biology, 18(6), 1607-1616.

Hidalgo, C., Sánchez, J., Sánchez, C., \& Saborío, M. T. (1995). Migración de Falconiformesen Costa Rica. HMANA Hawk Migration Studies, 21(1),10-13. 
Henderson, P. A., \& Seaby, R. M. (2006). Species, diversity and richness. Versión 4.0 Pisces Conservation Ltda. (Programa informático): Pennigton, Lyminton.

Magurran, A. E. (1988). Ecological diversity and its Measurement. (1 ${ }^{\text {a }}$. Ed.) Princeton University Press: New Jersey.

Martin, T. G., \& McIntyre, S. (2007). Impacts of Livestock Grazing and Tree Clearing on Birds of Woodland and Riparian Habitats. Conservation Biology, 21(2), 504-514.

Lampila, P., Monkkonen, M., \& Desrochers, A. (2005). Demographic Responses by Birds to Forest Fragmentation. Conservation Biology, 19(5), 1537-1546.

Lopez, H. (1998). Comparación de la avifauna de sotobosque en un paisaje fragmentado del pacífico central, Costa Rica. (Tesis de maestría inédita). PRMVS. Universidad Nacional, Heredia, Costa Rica.

Obando-Calderón, G., Chaves-Campos, J., Garrigues, R., Martínez-Salinas, A., Montoya, M., Ramirez, O., \& Zook, J. (2013). Boletín de la Asociación Ornitológica de Costa Rica. San José, Costa Rica. Zeledonia, 17, 2.

Petit, L. J., \& Petit, D. R. (2003). Evaluating the Importance of Human-Modified Lands for Neotropical Bird Conservation. Conservation Biology, 17(3), 687-694.

Pereira, A. I., \& Barrantes, G. (2009). Distribución y densidad de la avifauna de la Península de Osa, Costa Rica (1990-1991). Revista de Biología Tropical, 57(1), 323-332.

ProAmbiente. (1998). Evaluación Ecológica Rápida Corredor Biológico Talamanca Caribe. Asociación Nacional Pro Medio Ambiente y Recursos Naturales: San José, Costa Rica.
Ramírez, J. E. (2010). Diversidad de aves de habitats naturales y modificados en un paisaje de la Depresión Central de Chiapas, México. Revista de Biología Tropical, 58(1), 511-528.

Ralph, C. J. , Geupel, G. R., Pyle, P., Martin, T. E., \& De Sante, D. F. (1993). Manual de Métodos de Campo para el Monitoreo de Aves Terrestres. Informe Técnico General. PSW GTR 144. Estación de Investigaciones del Pacífico Suroeste, Servicio Forestal de EEUU. Departamento de Agricultura. Washington D.C.

Rodewald, P. G., \& Brittingham, M. C. (2007). Stopover habitat use by spring migrant landbirs: The roles of habitat structure, leaf development, and food availability. The Auk, 124(3), 1063-1074.

Sánchez, J.; Naoki, K., \&Zook, J. (1998). New Information About Costa Rican Birds. Ornitología Neotropical, 9, 99-102.

Sigel, B. J., Sherry, T. W., \& Young, B. E. (2006). Avian Community Response to Lowland Tropical Rainforest Isolation: 40 Years of Change at La Selva Biological Station, Costa Rica. Conservation Biology, 20(1), 111-121.

Stiles, F. G. (1983). Cambios altitudinales y estacionales en la avifauna de la vertiente atlántica de Costa Rica. I Simposio de Ornitología Neotropical (CLAZ, Perú 1983).

Stiles, F. G., \& Skutch, A. (1989). A guide to the Birds of Costa Rica. Cornell University: Ithaca, NewYork.

Stiles, F. G. (1991). Lista de Aves. pp. 441 455. En D. H. Janzen (ed). Historia Natural de Costa Rica. Editorial de la Universidad de Costa Rica. San José.

Zar, J. H. (1996). Biostatistical analysis. Prentice Hall. Nueva Jersey, EEUU. 
ANEXO 1

Listado General de especies de aves observadas entre Moín y Cahuita, Limón; Costa Rica. Tipos de hábitat: Pa (potrero arbolado), $\mathrm{Hr}$ (hábitat ribereño), Bi (bosque intervenido), Ca (cultivo arbolado), $\mathrm{BiHr}$ (bosque intervenido con hábitats ribereños), Cm (cultivos mixtos), B (bosque maduro), LT (latizal tacotal), P (potreros de gramíneas) y LCB (latizal de caña brava Gynerium sagittatum); según el estatus migratorio: Estatus/Status: Residente/Resident (R), Migratorio/Migrant (M), Especies Endémicas para Costa Rica y la región (END) (Obando et al. 2012).

\begin{tabular}{|c|c|c|c|c|c|c|c|c|c|c|c|c|}
\hline \multirow{2}{*}{ Especies } & \multicolumn{10}{|c|}{ Habitats } & \multirow{2}{*}{ Abundancia relativa } & \multirow{2}{*}{ Estatus migratorio } \\
\hline & B & $\mathrm{Bi}$ & $\mathrm{BIHr}$ & $\mathrm{Ca}$ & $\mathrm{Cm}$ & $\mathrm{Hr}$ & LCB & LT & $P$ & $\mathrm{~Pa}$ & & \\
\hline Psarocolius montezuma & & $x$ & $x$ & $x$ & $x$ & $x$ & & $x$ & $x$ & $x$ & 2,08 & $\mathrm{R}$ \\
\hline Ramphocelus passerinii & $x$ & $x$ & $x$ & $x$ & & $x$ & & $x$ & & $\mathrm{x}$ & 1,49 & $\mathrm{R}$ \\
\hline Oryzoborus funereus & $x$ & $x$ & $x$ & $x$ & $x$ & $x$ & & $x$ & & $x$ & 1,38 & $\mathrm{R}$ \\
\hline Pitangus sulphuratus & & $x$ & & $x$ & $x$ & $x$ & & & & $x$ & 0,89 & $\mathrm{R}$ \\
\hline Melanerpes pucherani & & $x$ & & $x$ & $x$ & $x$ & & $x$ & & $x$ & 0,83 & $\mathrm{R}$ \\
\hline Arremonops conirostris & & $x$ & & $x$ & $x$ & $x$ & & $x$ & & $x$ & 0,80 & $\mathrm{R}$ \\
\hline Ramphastos sulfuratus & & $x$ & $x$ & $x$ & & $x$ & & & & $x$ & 0,79 & $\mathrm{R}$ \\
\hline Aratinga nana & & $x$ & & & $x$ & $x$ & & & & $x$ & 0,73 & $\mathrm{R}$ \\
\hline Tangara larvata & & $x$ & $x$ & $x$ & & $x$ & & & & $x$ & 0,71 & $\mathrm{R}$ \\
\hline Euphonia luteicapilla & & $x$ & $x$ & $x$ & $x$ & $x$ & & & & $x$ & 0,50 & R-END \\
\hline Crotophaga sulcirostris & & & $x$ & $x$ & $x$ & & & $x$ & & $x$ & 0,50 & $\mathrm{R}$ \\
\hline Campylorhynchus zonatus & & $x$ & $x$ & $x$ & $x$ & $x$ & & & & $x$ & 0,45 & $\mathrm{R}$ \\
\hline Turdus grayi & $x$ & $x$ & $x$ & $x$ & $x$ & $x$ & & $x$ & & $x$ & 0,42 & $\mathrm{R}$ \\
\hline Xiphorhynchus susurrans & & $x$ & $x$ & $x$ & & $x$ & & & & $x$ & 0,42 & $\mathrm{R}$ \\
\hline Thraupis palmarum & $x$ & $x$ & & & & $x$ & & & & $x$ & 0,37 & $\mathrm{R}$ \\
\hline Tityra semifasciata & & $x$ & $x$ & $x$ & & $x$ & & $x$ & & $x$ & 0,36 & $\mathrm{R}$ \\
\hline Cantorchilus nigricapillus & & $x$ & $x$ & $x$ & $x$ & $x$ & $x$ & $x$ & & $x$ & 0,33 & $\mathrm{R}$ \\
\hline Cathartes aura & & $x$ & & $x$ & & $x$ & & & & $x$ & 0,33 & $\mathrm{R}, \mathrm{M}$ \\
\hline Patagioenas nigrirostris & & $x$ & & $x$ & $x$ & $x$ & & $x$ & & $x$ & 0,29 & $\mathrm{R}$ \\
\hline Saltator maximus & & $x$ & & $x$ & & $x$ & & & & $x$ & 0,29 & $\mathrm{R}$ \\
\hline Vireo olivaceus & & $x$ & & & & & & & & $x$ & 0,29 & M \\
\hline Myrmeciza exsul & & $x$ & $x$ & $x$ & & $\mathrm{x}$ & & & & & 0,29 & $\mathrm{R}$ \\
\hline Troglodytes aedon & $x$ & & & $x$ & $x$ & & & & $x$ & $x$ & 0,27 & $\mathrm{R}$ \\
\hline Tyrannus melancholicus & & & $x$ & $x$ & $x$ & $x$ & & & & $x$ & 0,24 & $\mathrm{R}$ \\
\hline Hirundo rustica & & & & & $x$ & $x$ & & & & $x$ & 0,23 & M \\
\hline Pteroglossus torquatus & & $x$ & & $x$ & $x$ & $x$ & & $x$ & & $x$ & 0,22 & $\mathrm{R}$ \\
\hline Setophaga pensylvanica & & $x$ & & $x$ & $x$ & $x$ & & & & $x$ & 0,22 & M \\
\hline Thraupis episcopus & $x$ & & $x$ & $x$ & & & & $x$ & & $x$ & 0,20 & $\mathrm{R}$ \\
\hline Todirostrum cinereum & & & & & $x$ & $x$ & & $x$ & & $x$ & 0,20 & $\mathrm{R}$ \\
\hline Tinamus major & & $x$ & & $x$ & & $x$ & $x$ & & & $\mathrm{x}$ & 0,20 & $\mathrm{R}$ \\
\hline Aratinga finschi & & & & & & & & & & $x$ & 0,20 & R-END \\
\hline Contopus cinereus & & $x$ & & & $x$ & $x$ & & & & $x$ & 0,19 & $\mathrm{R}$ \\
\hline Coragyps atratus & & & & & & & & & & $x$ & 0,19 & $\mathrm{R}$ \\
\hline Cacicus uropygialis & & $x$ & & $x$ & & & & & & $x$ & 0,18 & $\mathrm{R}$ \\
\hline Manacus candei & & $x$ & & & & $x$ & & & & $x$ & 0,18 & $\mathrm{R}$ \\
\hline Amazilia tzacatl & & $x$ & & & $x$ & $x$ & & & & $x$ & 0,17 & $\mathrm{R}$ \\
\hline Trogon caligatus & & & & $x$ & & $x$ & & & & $x$ & 0,15 & $\mathrm{R}$ \\
\hline Volatinia jacarina & & & & & & & & & & $x$ & 0,14 & $\mathrm{R}$ \\
\hline Tityra inquisitor & & & & $x$ & $x$ & $x$ & & & & $x$ & 0,11 & $\mathrm{R}$ \\
\hline Polioptila plumbea & & & & $x$ & $x$ & & & & & $x$ & 0,11 & $\mathrm{R}$ \\
\hline Chondrohierax uncinatus & & $x$ & & & & $x$ & & & & $x$ & 0,11 & $\mathrm{R}$ \\
\hline
\end{tabular}


ANEXO 1 (Continuación)

\begin{tabular}{|c|c|c|c|c|c|c|c|c|c|c|c|c|}
\hline \multirow{2}{*}{ Especies } & \multicolumn{10}{|c|}{ Habitats } & \multirow{2}{*}{ Abundancia relativa } & \multirow{2}{*}{ Estatus migratorio } \\
\hline & B & $\mathrm{Bi}$ & $\mathrm{BIHr}$ & $\mathrm{Ca}$ & $\mathrm{Cm}$ & $\mathrm{Hr}$ & LCB & LT & $P$ & $\mathrm{~Pa}$ & & \\
\hline Icterus mesomelas & & & & $x$ & $x$ & & & & & $\mathrm{x}$ & 0,11 & $\mathrm{R}$ \\
\hline Cyanocorax affinis & & & & & & $x$ & & & & $x$ & 0,10 & $\mathrm{R}$ \\
\hline Pachyramphus cinnamomeus & & & & $\mathrm{x}$ & $\mathrm{x}$ & & & & & $\mathrm{x}$ & 0,10 & $\mathrm{R}$ \\
\hline Patagioenas cayennensis & & & $x$ & $x$ & $x$ & $x$ & & & & $x$ & 0,10 & $\mathrm{R}$ \\
\hline Myiarchus crinitus & & $x$ & $x$ & $x$ & & & & & & $x$ & 0,09 & M \\
\hline Tachyphonus rufus & & & & $x$ & $x$ & $x$ & & & & $\mathrm{x}$ & 0,09 & $\mathrm{R}$ \\
\hline Caryothraustes poliogaster & $x$ & & & & $x$ & $x$ & & & & & 0,08 & $\mathrm{R}$ \\
\hline Tachyphonus delatrii & & & & & & & & & & & 0,08 & $\mathrm{R}$ \\
\hline Colonia colonus & & & & & $x$ & $x$ & & & & $\mathrm{x}$ & 0,08 & $\mathrm{R}$ \\
\hline Euphonia gouldi & & & & $x$ & & & & & & & 0,08 & $\mathrm{R}$ \\
\hline Icterus galbula & & & & & & & & & & $\mathrm{x}$ & 0,08 & M \\
\hline Aramides cajaneus & & $x$ & $x$ & & $x$ & $x$ & & $x$ & & & 0,08 & $\mathrm{R}$ \\
\hline Oreothlypis peregrina & & & & & & $x$ & & & & $\mathrm{x}$ & 0,06 & M \\
\hline Chloroceryle americana & & & $x$ & & & $x$ & & & & & 0,06 & $\mathrm{R}$ \\
\hline Hylophilus decurtatus & & & & $x$ & & & & & & & 0,06 & $\mathrm{R}$ \\
\hline Crypturellus boucardi & & $x$ & & & & & & & & $x$ & 0,06 & $\mathrm{R}$ \\
\hline Piaya cayana & & & & $x$ & & & & & $x$ & $x$ & 0,05 & $\mathrm{R}$ \\
\hline Chlorothraupis carmioli & & & & & & & & & & $\mathrm{x}$ & 0,05 & $\mathrm{R}$ \\
\hline Deconychura longicauda & & $x$ & & & & & & & & & 0,05 & $\mathrm{R}$ \\
\hline Pionus senilis & & $x$ & & & & & & & & $x$ & 0,05 & R \\
\hline Synallaxis brachyura & & & & & & $x$ & & & & & 0,05 & $\mathrm{R}$ \\
\hline Molothrus aeneus & & & & & & & & & & $x$ & 0,05 & $\mathrm{R}$ \\
\hline Riparia riparia & & & $x$ & & & & & & & & 0,04 & M \\
\hline Notharchus tectus & & & & & & & & & & $x$ & 0,04 & $\mathrm{R}$ \\
\hline Egretta thula & & & $x$ & & & & & & & $x$ & 0,04 & $\mathrm{R}, \mathrm{M}$ \\
\hline Nothocercus bonapartei & & & & & & $x$ & & $x$ & & $x$ & 0,04 & $\mathrm{R}$ \\
\hline Trogon massena & & $x$ & & & & & & & & & 0,04 & $\mathrm{R}$ \\
\hline Cercomacra tyrannina & & & & & & $x$ & & & & & 0,04 & $\mathrm{R}$ \\
\hline Troglodytes aedon & $x$ & & & & & & & & & & 0,04 & R \\
\hline Bubulcus ibis & & & & & & & & $x$ & & & 0,03 & $\mathrm{R}, \mathrm{M}$ \\
\hline Pheugopedius atrogularis & & & & & & $x$ & & & & & 0,03 & R-END \\
\hline Chlorophanes spiza & & & & & & & & & & & 0,03 & R \\
\hline Chloroceryle inda & & & & & & $x$ & & & & & 0,03 & $\mathrm{R}$ \\
\hline Ortalis cinereiceps & & & & & & $x$ & & & & & 0,03 & R \\
\hline Phaethornis striigularis & $\mathrm{x}$ & $\mathrm{x}$ & & & & & & & & & 0,03 & $\mathrm{R}$ \\
\hline Thamnophylus atrinucha & & & & & & & & & & & 0,03 & $\mathrm{R}$ \\
\hline Saltator atriceps & & $x$ & & & $x$ & & & $x$ & & & 0,03 & $\mathrm{R}$ \\
\hline Notharchus hyperrhynchus & & & & $x$ & & & & & & & 0,03 & $\mathrm{R}$ \\
\hline Leptotila verreauxi & & & & & & & & $x$ & & $x$ & 0,03 & $\mathrm{R}$ \\
\hline Catorchilus modestus & & & & & $x$ & $x$ & & & & & 0,03 & $\mathrm{R}$ \\
\hline Anthracothorax prevostii & & & & & & $x$ & & & & $x$ & 0,03 & $\mathrm{R}$ \\
\hline Traupis palmarum & & $x$ & & & & & & & & & 0,03 & $\mathrm{R}$ \\
\hline Phaethornis longirostris & & $x$ & & & & & & & & $x$ & 0,03 & R \\
\hline Setophaga coronata & & & & & & & & & & $x$ & 0,03 & M \\
\hline Taraba major & & $x$ & & & & & & & & $\mathrm{x}$ & 0,03 & R \\
\hline Contopus sordidulus & & & & & & $\mathrm{x}$ & & & & $\mathrm{x}$ & 0,03 & $M, R$ \\
\hline
\end{tabular}


ANEXO 1 (Continuación)

\begin{tabular}{|c|c|c|c|c|c|c|c|c|c|c|c|c|}
\hline \multirow{2}{*}{ Especies } & \multicolumn{10}{|c|}{ Habitats } & \multirow{2}{*}{ Abundancia relativa } & \multirow{2}{*}{ Estatus migratorio } \\
\hline & B & $\mathrm{Bi}$ & $\mathrm{BIHr}$ & $\mathrm{Ca}$ & $\mathrm{Cm}$ & $\mathrm{Hr}$ & LCB & LT & $P$ & $\mathrm{~Pa}$ & & \\
\hline Carpodectes nitidus & & $x$ & & & & $x$ & & & & & 0,03 & R-END \\
\hline Attila spadiceus & & & & & & $\mathrm{x}$ & & & & & 0,03 & $\mathrm{R}$ \\
\hline Sporophila torqueola & & & & & & & & & & $\mathrm{x}$ & 0,03 & $\mathrm{R}$ \\
\hline Sittasomus griseicapillus & & $x$ & & & & & & & & & 0,01 & $\mathrm{R}$ \\
\hline Trogon rufus & & & & & $x$ & & & & & $x$ & 0,01 & $\mathrm{R}$ \\
\hline Poecilotriccus sylvia & & & & & & & & & & $x$ & 0,01 & $\mathrm{R}$ \\
\hline Myozetetes similis & & & & & & & $x$ & & & & 0,01 & $\mathrm{R}$ \\
\hline Megarhynchus pitangua & & & & & & & & & & $x$ & 0,01 & $\mathrm{R}$ \\
\hline Onychorhynchus coronatus & & & $x$ & & & & & & & & 0,01 & $\mathrm{R}$ \\
\hline Buteogallus urubitinga & & & & & & & & & & & 0,01 & $\mathrm{R}$ \\
\hline Egretta caerulea & & & $x$ & & & & & & & & 0,01 & $\mathrm{R}, \mathrm{M}$ \\
\hline Leptotila cassini & & & & & & & & & & $x$ & 0,01 & $\mathrm{R}$ \\
\hline Crypturellus soui & & & & & & $x$ & & & & & 0,01 & $\mathrm{R}$ \\
\hline Catharus fuscescens & & & & & & $x$ & & & & & 0,01 & M \\
\hline Electron platyrhynchum & & $x$ & & & & & & & & & 0,01 & $\mathrm{R}$ \\
\hline Parkesia noveboracensis & & & $x$ & & & & & & & & 0,01 & M \\
\hline Tapera naevia & & & & & & & & & $x$ & & 0,01 & $\mathrm{R}$ \\
\hline Coereba flaveola & & & & & & & & & & $x$ & 0,01 & $\mathrm{R}$ \\
\hline Cyanerpes cyaneus & $x$ & & & & & & & & & & 0,01 & $\mathrm{R}$ \\
\hline Arremon aurantirostris & & & & & & $x$ & & & & & 0,01 & $\mathrm{R}$ \\
\hline Terenotriccus erythrurus & & & & $x$ & & & & & & & 0,01 & $\mathrm{R}$ \\
\hline Setophaga fusca & & & & & & & & & & $x$ & 0,01 & M \\
\hline Piranga bidentata & & & & & $x$ & & & & & & 0,01 & $\mathrm{R}$ \\
\hline Baryphthengus martii & & $x$ & & & & & & & & & 0,01 & $\mathrm{R}$ \\
\hline Piranga rubra & & & & & & & & & & $x$ & 0,01 & M \\
\hline Pachyramphus aglaiae & & & & & & & & & & & 0,01 & $\mathrm{R}$ \\
\hline Cyanerpes lucidus & & & & & & & & & & $x$ & 0,01 & R \\
\hline Setophaga petechia & & & & & & & & & & $x$ & 0,01 & $R, M$ \\
\hline Geothlypis formosa & & & & & & & & & & $x$ & 0,01 & M \\
\hline Tyrannus tyrannus & & & & & & & & & & $x$ & 0,01 & M \\
\hline Geothlypis philadelphia & & & & & & & & & $x$ & & 0,01 & M \\
\hline Micrastur ruficollis & & & & & & & & & & $x$ & 0,01 & $\mathrm{R}$ \\
\hline Herpetotheres cachinnans & & & $x$ & & & & & & & & 0,01 & $\mathrm{R}$ \\
\hline Conopias albovittatus & & $x$ & & & & & & $x$ & & & 0,01 & R \\
\hline Myiozetetes granadensis & & & & $x$ & & & & & & & 0,01 & $\mathrm{R}$ \\
\hline Dives dives & & & & & & & & & & $x$ & 0,01 & $\mathrm{R}$ \\
\hline
\end{tabular}

